\title{
V021 4D SEISMIC REPEATABILITY OVER THE GULLFAKS FIELD - SOURCE AND RECEIVER POSITIONING ISSUES
}

\author{
JAMES KEGGIN and BJØRN OLAV EKREN \\ Statoil and BPR \& D Alliance, Rotvoll, Postuttak, 7005 Trondheim, Norway
}

\section{Introduction}

Reservoir monitoring using repeat 3D surveys is potentially a very powerful tool for identifying remaining reserves in existing and new fields. Today the method is in its infancy and is, as yet, not fully proven. The success of the method will depend, amongst other factors, upon the quality and repeatability of our 3D datasets. The challenge for the geophysicist is to ensure that the change in seismic response due to changes in the reservoir rock properties is greater than the inherent errors and lack of repeatability of our seismic data. In a perfect world, repeat seismic data would be acquired with identical equipment under identical environmental conditions. Since these ideal requirements are never met, it is essential that we understand the magnitude of any errors that might be introduced in our data. This study examines the effect of navigation errors and the effect of mis-matched source-receiver azimuths on marine seismic repeatability. The work was carried out as part of a reservoir monitoring project on the Gullfaks field in the Norwegian North Sea. 3D datasets from 1985 and 1995 were used.

\section{Navigation errors}

If we are to use the 4D seismic method on our existing fields, we will almost certainly be using data from existing 3D surveys. Although today's positioning systems are usually accurate to a few metres, significant errors are to he found on older datasets. Before the advent of GPS, active tailbuoys and acoustic positioning systems in the late 1980 's, errors of over $100 \mathrm{~m}$ on the far offsets and $20 \mathrm{~m}$ on the near offsets were common.

To examine the effect of positioning errors on pre-stack seismic data, we extracted traces from a 1985 and a 1995 3D dataset that had nearly identical source and receiver locations. These traces were from an area where we expected no change in rock properties between 1985 and 1995. Figure 1 shows the near offset cable position of a matching pair of shot records (the solid line ' 85 port cable matches the hashed line ' 95 inner port cable). Corresponding source and receiver positions are within $10 \mathrm{~m}$ of each other. The traces from these partial shot records are shown in Figure 2. The 1985 shot was then subtracted from the adjacent 1995 port side shots to illustrate mispositioning. The surrounding 1995 shot records are shown in Figure 3 and the resulting difference data are shown in Figure 4. The amplitudes of the difference data were measured over a large window and displayed graphically in Figures 5 and 6 . It is clear that the best match is occurring where expected. This suggests that both navigation datasets are free from large errors. It is also comforting to see that the two datasets are very similar. Most striking of all is the sensitivity of the difference plot to the simulated mispositioning of the 1985 data. A $50 \mathrm{~m}$ bulk shift in position produces a difference amplitude of the same magnitude as the input. Similar experiments on far offset data showed cross-line positional errors of over $50 \mathrm{~m}$. Given that errors of $50 \mathrm{~m}$ and over are known to exist, it will be impossible to produce meaningful pre-stack difference data that can be processed and interpreted. This sensitivity to positioning error will be directly related to geological complexity - these data were shot over an area of rapidly varying geology. Clearly, in areas of simple layercake geology, the difference surface shown in Figure 5 will probably have a much broader minimum. 


\section{Azimuthal variations between two datasets.}

Hidden in every post stack volume and every fold of coverage map are the shot-receiver azimuths of the pre-stack traces. Although a pair of repeated CMPs may have identical fold and offset distribution, they will almost certainly contain traces with different azimuths (assuming the data has been recorded with surface towed cables). The key questions are: How do mismatched azimuths effect repeatability? How big are these effects ? and can these effects be compensated for in processing? All of these issues will be discussed using data before and after stack. An extreme case of this problem is shown in Figure 7 where 1985 data with very small feathering angles are compared with undershoot data from 1995. Since our repeat seismic surveys will be shot over producing fields, the undershooting of surface obstructions will cause widespread problems. The degree to which we can correct for mis-matched azimuths will be a key factor when designing acquisition techniques for 4D seismic. If we use fixed receiver techniques, it will be possible to repeat the location of shot and receiver as well as CMP position. Fixed receiver acquisition schemes (seabed cable or vertical cable for example) will be much less troubled by surface obstructions than the conventional surface tow method.

\section{Conclusions}

1. Positioning errors cause significant errors on pre-stack difference data. These large errors suggest that pre-stack difference data cannot be used in a meaningful way on older 3D datasets.

2. Azimuthal differences cause significant errors on pre-stack difference data. These are difficult to eliminate during processing.

3. Although the pre-stack differenced data are extremely sensitive to source and receiver position, it should still be possible to draw sensible conclusions from repeat datasets by using other interpretation methods. This point has been well illustrated in several case histories (e.g Watts et. al. 1995).

4. For this datset, the repeatability errors introduced as a result of mis-matched source and receiver coordinates are greater that those introduced by differing acquisition hardware (source signature, cable response, recording systems).

5. The problems of inaccurate positioning and azimuth mis-match will be most acute for surface towed cable data. Fixed receiver techniques (seabed cable for example) should not suffer from these problems provided that the source and receiver positions are accurately measured. If other problems such as stronger multiples, asymmetric raypaths, coupling effects and adequate receiver coverage can be overcome, the fixed receiver type of acquisition looks very attractive for repeat $3 \mathrm{D}$ surveys.

\section{Acknowledgments.}

Thanks to Ian Jack, Andy Morton and Neil Philip for their advice, criticism and practical help. We thank the partnership of Statoil, Saga and Norsk Hydro for releasing these data for publication.

\section{References:}

Manin, Boucquaert, Reghnaudin, Regnault \& Thevenot, Recent developments in source and receiver positioning, First Break Vol 6, No. 6, June 1988.

Mark Houston, Cable positioning using compesses, tailbuoys and acoustic devices, 53rd Mtg EAEG abstracts 1991.

Manin, Can we improve binning from the study of seismic images?, 48th Mtg EAEG abstracts 1986.

Watts, Jizba, Gawith, Gutteridge, Reservoir monitoring of the Magnus field through 4D time-lapse seismic analysis, Presented 7th EAPG annual conference June 1995.

David E Lumley, Seismic time-lapse monitoring of subsurface fluid flow, PhD Thesis, Stanford Exploration Project, Dec 1995. 